\title{
Análise da diversidade de verbos enunciados na fala espontânea de pré-escolares brasileiros***
}

\author{
Verb diversity analysis in the spontaneous speech of Brazilian \\ preschoolers
}

Debora Maria Befi-Lopes*

Ana Manhani Cáceres**

*Fonoaudióloga. Livre-Docente do Curso de Fonoaudiologia da Faculdade de Medicina da Universidade de São Paulo. Professora Associada do Departamento de Fisioterapia, Fonoaudiologia e Terapia Ocupacional da Faculdade de Medicina da Universidade de São Paulo. Endereço para correspondência: Rua Cipotânea, 51 - São Paulo - SP - CEP 05360-160 (dmblopes@usp.br).

**Fonoaudióloga. Colaboradora do Laboratório de Investigação Fonoaudiológica em Desenvolvimento da Linguagem e suas Alterações, do Curso de Fonoaudiologia, Departamento de Fisioterapia, Fonoaudiologia e Terapia Ocupacional da Faculdade de Medicina da Universidade de São Paulo.

***Trabalho Realizado no Laboratório de Investigação Fonoaudiológica em Desenvolvimento da Linguagem e suas Alterações, do Curso de

Fonoaudiologia, Departamento de Fisioterapia, Fonoaudiologia e Terapia Ocupacional da Faculdade de Medicina da Universidade de São Paulo.

Artigo Original de Pesquisa

Artigo Submetido a Avaliação por Pares

Conflito de Interesse: não

Recebido em 29.07.2009.

Revisado em 05.01.2010.

Aceito para Publicação em 01.02.2010.

\section{Abstract}

Background: verb acquisition. Aim: to verify quantitative and qualitative verb diversity in the spontaneous speech of Brazilian Portuguese-speaking preschoolers and verb acquisition from 2 to 4 years of age. Method: participants were sixty preschoolers equally matched on gender and age, divided into three groups: GI (2-year-old children), GII (3-year-old children) and GIII (4-year-old children). Spontaneous speech samples obtained through playing interactions, in educational contexts, were collected, and verb productions were listed. Results: children used 167 different verbs. The quantitative analysis indicated significant statistical differences between all groups $(\mathrm{p}<.001)$, with gradual increase of verb usage from 2 to 4 years of age. However, no statistical difference $(p=.956)$ was observed between genders. Conclusion: preschoolers improved their use of verbs during the initial stages of language acquisition, irrespective of their gender.

Key Words: Language Development; Child Language; Vocabulary, Preschool.

\section{Resumo}

Tema: aquisição de verbos. Objetivos: verificar a diversidade quantitativa e qualitativa dos verbos enunciados por pré-escolares falantes do Português Brasileiro, bem como sua evolução dos 2 aos 4 anos de idade. Método: participaram do estudo sessenta pré-escolares divididos em três grupos pareados quanto ao gênero e de acordo com a faixa etária, a saber, GI (2 anos), GII (3 anos) e GIII (4 anos). Foram coletadas amostras de fala, obtidas em contexto educacional por meio de interação lúdica, a partir das quais foi empreendido um levantamento dos verbos empregados. Resultados: foram enunciados 168 verbos distintos, dos quais a análise da quantidade indicou diferenças estatísticas significantes entre os grupos (p-valor $<0,001$ ), com um aumento gradual no uso de verbos dos dois aos quatro anos, com diferenças entre todos os grupos. Todavia, não há diferença significante ( $\mathrm{p}$ - valor 0,956) entre os gêneros. Conclusão: os préescolares estudados aprimoraram o uso de verbos ao longo desta fase inicial de aquisição da linguagem, independente do gênero a que pertencem.

Palavras-Chave: Desenvolvimento da Linguagem; Linguagem Infantil; Vocabulário; Pré-escolar. 


\section{Introdução}

Estudos em diversas línguas apontam o verbo como um elemento que favorece o desenvolvimento gramatical, visto que expressa ações e processos e tem por função principal atuar como predicado ${ }^{1-4}$.

Um fator interessante com relação à sua aquisição consiste no privilégio que a fala materna tende a dar aos substantivos, pois os pais influenciam seus filhos a nomear objetos muito mais do que nomear ações, pois os verbos costumam estar mais relacionados a comportamentos não verbais do que a comportamentos verbais ${ }^{5}$.

Apesar de sua variedade semântica e gramatical, durante a expansão do vocabulário, há um aumento na gama de verbos empregados ${ }^{6-7}$. Destaca-se que as informações sintáticas favorecem a interpretação de sentenças com um verbo desconhecido e, conseqüentemente, o aprendizado de seu significado. Assim, a informação estrutural, aliada à informação contextual, é fundamental para a fixação do significado de verbos novos pela criança ${ }^{8-9}$.

Todavia, mesmo entre os verbos, são notadas diferenças quanto à aquisição, por exemplo, verbos com referência a ações físicas (como puxar) são identificados com maior facilidade do que aqueles que se referem a estados mentais (como querer) ${ }^{10}$. Além disto, quanto mais um verbo for estimulado, mais frequentemente e com maior flexibilidade ele aparecerá na fala da criança, o que indica que o refinamento de seu conhecimento ocorre por meio do uso ${ }^{11-16}$.

O objetivo deste estudo é verificar a diversidade quantitativa e qualitativa dos verbos enunciados por pré-escolares falantes do Português Brasileiro, bem como sua evolução dos 2 aos 4 anos de idade.

\section{Método}

O estudo foi autorizado pela Comissão de Ética para Análise de Projetos de Pesquisa da Diretoria Clínica do Hospital das Clínicas da Faculdade de Medicina da Universidade de São Paulo sob número 360/01. Previamente à coleta de dados, os pais foram esclarecidos a respeito dos objetivos do estudo e da forma de obtenção dos dados e então assinaram do "Termo de Consentimento Livre e Esclarecido".

Todos os sujeitos estavam matriculados em creches do município de São Paulo, localizadas na zona norte da referida cidade. As instituições mantinham convênio com a prefeitura e atendiam crianças de classe econômica média-baixa e baixa. Com o objetivo de caracterizar o desempenho de linguagem destas foi realizada uma triagem fonoaudiológica que verificava as habilidades de vocabulário, fonologia, produção e compreensão de sentenças ${ }^{17}$. Após a realização da triagem, foram selecionadas 60 crianças, em idade pré-escolar, com desenvolvimento normal de linguagem.

Os sujeitos foram divididos em três grupos conforme a faixa etária a qual pertenciam, observando que cada grupo deveria conter vinte sujeitos, sendo dez do sexo feminino e dez do masculino. O Grupo 1 (GI) comportou a faixa etária de 2:0 a 2:11 anos, com média de 2 anos e 5 meses; o Grupo 2 (GII) comportou a faixa etária de 3:0 a 3:11 anos, com média de 3 anos e 4 meses; e o Grupo 3(GIII) comportou a faixa etária de 4:0 a 4:11 anos, com média de 4 anos e 4 meses.

Com base nas faixas etárias estudadas e a necessidade de obtenção de fala espontânea, a amostra de fala foi coletada a partir de situação de interação lúdica com brinquedos, a saber: miniatura de fazenda com animais, meios de transporte, alimentos, utensílios de cozinha e dois bonecos. Além destes materiais, foram utilizados uma filmadora VHS, fitas de vídeo, um gravador e fitas cassete.

Para a coleta dos dados, uma sala foi preparada com uma filmadora posicionada sob um tripé e um gravador cassete ao lado da criança e os brinquedos foram dispostos em um tapete da seguinte forma: os utensílios de cozinha ficavam localizados à frente e no centro, os animais e fazendinha à direita e os meios de transporte e bonecos à esquerda.

Cada uma das crianças era solicitada a se retirar de sua sala de aula e, então, era levada para esta outra sala. A criança era encorajada a interagir com a pesquisadora por um período de 30 minutos, no qual eram realizadas perguntas abertas com o objetivo de propiciar a melhor situação de iniciativa por parte da criança. Deve-se ressaltar que os brinquedos utilizados propiciaram uma situação de comunicação efetiva, favorecendo comentários e relatos por parte das crianças, inclusive das mais novas.

Após a coleta dos dados, foi realizada a transcrição das amostras de fala. Para cada transcrição, o procedimento adotado foi: primeiramente, a pesquisadora assistia toda a gravação sem realizar nenhuma anotação, para observar o desempenho geral da criança. Num segundo momento, ela assistia a gravação realizando a transcrição. A amostra de fala foi dividida em segmentos (cada enunciado produzido pela criança), até que se chegasse ao número esperado de 100 segmentos $^{18}$. Quando este número foi atingido, o restante da transcrição foi desprezado. Para que se pudesse perceber e dividir corretamente cada segmento, pelo menos um dos critérios deveria ocorrer: mudança de assunto por parte da criança, mudança do foco de atenção da criança ou interrupção do terapeuta. Outro critério adotado na transcrição foi utilizar segmentos 
completamente transcritos, nenhum com quebras. Todas as repetições exatas de segmentos foram incluídas. As disfluências, como as repetições de palavra, foram pontuadas uma única vez. Em poucos casos, quando as palavras foram produzidas para dar ênfase, cada ocorrência foi contabilizada ${ }^{19}$.

Após a transcrição de todas as fitas, foi composta a lista de todos os verbos produzidos por cada sujeito e contabilizada a quantidade de vezes em que cada um ocorreu na amostra de fala de cada sujeito. A seguir, estes foram contados, agora eliminando os repetidos, para que se pudesse obter a diversidade de verbos que cada sujeito apresentou. Por fim, foi procedido um levantamento de todos os verbos produzidos neste corpus, considerando o total de sujeitos que utilizou cada um deles. Vale ressaltar que ovalormáximo de ocorrênciapossível equivaleria a 60 - número de sujeitos do estudo.

Para a análise estatística dos resultados foramutilizados os seguintes testes: teste-t pareado e independente e a análise de variância(ANOVA) para as comparações entre os gêneros e os grupos para cada uma das variáveis, supondo-se igualdade de variância e distribuição normal. Para as múltiplas comparações foi utilizado o testede Tukey. O nível de significância adotado foi de 5\%.

\section{Resultados}

A Tabela 1 apresenta a análise estatística descritiva da quantidade de verbos enunciados de acordo com o grupo. A ANOVA indicou haver diferenças significantes entre os grupos (p-valor $<0,001)$. O teste de Tukey aponta um aumento gradual no uso de verbos dos dois aos quatro anos, com diferenças entre todos os grupos.

TABELA 1. Estatística descritiva da produção de verbos - análise da diversidade por grupo.

\begin{tabular}{c|c|c|c}
\hline Grupo & Média & Mediana & Desvio Padrão \\
\hline GI & 22,25 & 23,00 & 3,88 \\
GII & 26,80 & 28,00 & 4,62 \\
GIII & 34,70 & 35,50 & 5,71 \\
\hline
\end{tabular}

GI = grupo com 2 anos, GII = grupo com 3 anos, GIII = grupo com 4 anos.

TABELA 2. Estatística descritiva da produção de verbos - análise da diversidade por gênero.

\begin{tabular}{c|c|c|c}
\hline Sexo & Média & Mediana & Desvio Padrão \\
\hline feminino & 27,87 & 26,00 & 7,58 \\
masculino & 27,97 & 28,00 & 6,52 \\
\hline
\end{tabular}

$\mathrm{GI}=$ grupo com 2 anos, GII = grupo com 3 anos, GIII = grupo com 4 anos.
Na Tabela 2 são observados os valores da estatística descritiva com a comparação do desempenho entre os gêneros. A ANOVA apontou não indicou diferença significante (p-valor 0,956).

Para apresentar a distribuição dos 168 verbos observados neste estudo, estes foram divididos em intervalos de acordo com o número de sujeitos que os utilizaram (em ordem decrescente):

. 50 a 60 sujeitos: 6 verbos [estar, fazer, ir, olhar, ser, ter]; . 40 a 49 sujeitos: 6 verbos [querer, pegar, por, dar, poder, comer];

. 30 a 39 sujeitos: 3 verbos [cair, ficar, saber];

. 20 a 29 sujeitos: 10 verbos [dormir, tomar, deixar, gostar, tirar, fechar, ver, colocar, abrir, morder];

. 10 a 19 sujeitos: 24 verbos [caber, sair, achar, brincar, cortar, levar, quebrar, esperar, acabar, ajudar, esquentar, guardar, conseguir, vir, andar, arrumar, bater, chorar, fritar, lavar, chamar, chegar, comprar, passar];

. 02 a 09 sujeitos: 62 verbos [acordar, entrar, ligar, mexer, queimar, dirigir, doer, esquecer, machucar, montar, beber, correr, cozinhar, falar, passear, segurar, subir, trabalhar, andar, cuidar, derrubar, desligar, morar, trazer, botar, ganhar, jogar, mamar, morrer, sentar, deitar, descer, tampar, apertar, cobrir, consertar, esconder, esfriar, faltar, gritar, parar, prender, puxar, sarar, virar, voltar, almoçar, chover, conhecer, contar, destruir, escovar, grudar, limpar, matar, pensar, procurar, pular, secar, soltar, sumir, usar];

. apenas 01 sujeito: 57 verbos [acender, acontecer, acudir, adorar, apagar, apostar, arranhar, assistir, assoprar, assustar, atirar, atropelar, beliscar, brigar, buscar, cantar, carregar, chupar, conversar, dançar, derramar, desculpar, dizer, dobrar, encontrar, entortar, enxugar, errar, escolher, escorregar, espirrar, estudar, fingir, fugir, incomodar, jantar, juntar, lotar, lutar, mandar, melhorar, mijar, misturar, mostrar, nanar, nascer, ouvir, partir, precisar, rodar, roubar, sangrar, separar, servir, terminar, transformar, vender].

A Tabela 3 apresenta os dez verbos mais frequentes em cada um dos grupos.

\section{Discussão}

Com relação à diversidade verbal, os grupos se diferenciam, ocorrendo um aumento gradativo de GI a GIII, o que confirma que os verbos, apesar de começarem a ser empregados por volta dos dois anos de idade, tem seu uso aperfeiçoado entre os três a quatro anos de idade $\mathrm{e}^{1-8,14}$.

A aquisição dos verbos demonstrou não ser afetada pelo gênero, uma vez que não houve diferença estatisticamente significante. Assim, temos evidências que reforçam que as principais influências neste processo 
são tanto o ambiente comunicativo, quanto a condição biológica para desenvolver linguagem $1^{4-16,19}$.

A variedade de verbos utilizados confirma que o aprimoramento do desenvolvimento gramatical não se restringe à quantidade, mas engloba a variedade visto que a expansão do vocabulário não se restringe aos substantivos ${ }^{6-8,16,19}$.

Os verbos contidos na Tabela 3 evidenciam que os mais recorrentes nos três grupos são essencialmente os mesmos. Tal fato está de acordo com a literatura internacional que aponta que a familiaridade é um aspecto fundamental, pois está intrinsecamente relacionado à freqüência dos estímulos, o que favorece tanto uma maior recorrência de seu uso pela criança, quanto o uso em contextos diversificados ${ }^{12}$.

Em conformidade com outros estudos ${ }^{10-13}$, nota-se que dentre estes há uma leve disparidade entre os verbos que designam ações físicas e aqueles que designam estados mentais, onde os primeiros são mais frequentes.

\section{Conclusão}

Os resultados indicaram que os pré-escolares estudados aprimoraram o uso de verbos ao longo desta fase inicial de aquisição da linguagem, independente do gênero a que pertencem. Tal aprimoramento fica

\section{Referências Bibliográficas}

1. Marshall J. Noun-verb dissociations - evidence from acquisition and developmental and acquired impairments. J Neurolinguistics. 2003;16:67-84.

2. Skipp A, Windfuhr KL, Conti-Ramsden G. Children's grammatical categories of verb and noun: a comparative look at children with specific language impairment (SLI) and normal language (NL). Int J Lang Comm Dis. 2002;37:253-71.

3. Demuth K, Machobane M, Moloi F. Rules and construction effects in learning the argument struture of verbs. J Child Lang. 2003;30:797-821.

4. Devescovi A, Caselli MC, Marchione D, Pasqualetti P, Reilly J, Bates E. A cross linguistic study of the relationship between grammar and lexical development. J Child Lang. 2005;32:759-86

5. Goldfield BA. Nouns before verbs in comprehension vs. production: the view from pragmatics. J Child Lang. 2000; 27:501-20.

6. Ogura T, Dale PS, Yamashita Y, Murase T, Mahieu A. The use of nouns and verbs by Japanese children and their caregivers in book-reading and toy-playing contexts. J Child Lang. 2006;33:1-29.

8. Befi-Lopes DM, Cáceres AM, Araújo K. Aquisição de verbos em pré-escolares falantes do Português Brasileiro. Rev CEFAC. 2007;9:444-52.

9. Folli R, Harley H. What language says about the psychology of events. Trends Cogn Sci. 2006;10:91-2.
TABELA 3. Lista dos dez verbos mais freqüentes por grupo.

\begin{tabular}{ccc}
\hline GI $[\mathrm{n}=88]$ & GII $[\mathrm{n}=102]$ & GIII $[\mathrm{n}=147]$ \\
\hline $\begin{array}{c}\text { olhar, ser, estar, dar, ir, } \\
\text { querer, poder, ter, pegar, estar, fazer, pegar, } \\
\text { cair }\end{array}$ & $\begin{array}{c}\text { ser, olhar, ter, por, comer, fazer, estar, ir, } \\
\text { querer }\end{array}$ & $\begin{array}{c}\text { ser, olhar, poder, ficar, } \\
\text { comer }\end{array}$ \\
\hline
\end{tabular}

GI = grupo com 2 anos, GII = grupo com 3 anos, GIII = grupo com 4 anos, $\mathrm{n}=$ número de verbos distintos produzidos no grupo.

Pela observação dos verbos deste estudo podese notar a riqueza de combinações presentes na língua, bem como a criatividade permitida ao falante dentro de uma língua ${ }^{14-15}$, pois apesar de submetidos à mesma situação de comunicação, cada participante do presente estudo foi capaz de utilizar sentenças com verbos distintos para expressar de forma mais fiel suas intenções ${ }^{9}$.

evidente tanto pelo aumento da média de verbos utilizados por cada sujeito, quanto pela maior diversidade de verbos empregados conforme a faixa etária.

10. Gertner Y, Fisher C, Eisengart J. Learning words and rules: abstract knowledge of word order in early sentence comprehension. Psychol Sci. 2006;17(8):684-91.

11. Ambalu D, Chiat S, Pring T. When is it best to hear a verb? the effects of the timing and focus of verb models on children's learning of verbs. J Child Lang. 1997;24:25-34.

12. Naigles LR, Hoff-Ginsberg E. Why are some verbs learned before other verbs? Effects of input frequency and structure on children's early verb use. J Child Lang. 1998; 25:95-120.

13. Gillette J, Gleitman H, Gleitman L, Lederer A. Human simulations of vocabulary learning. Cognition 1999; 73:135-76.

14. Tomasello M. The item-based nature of children's early syntactic development. Trends Cogn Sci. 2000;4:156-63.

15. Keren-Portnoy T. Facilitation and practice in verb acquisition. J Child Lang. 2006;33:487-518.

16. Befi-Lopes DM, Cáceres AM. Verificação da morfologia verbal em pré-escolares falantes do Português Brasileiro. Rev Soc Bras Fonoaudiol. [prelo].

17. Fluharty NB. Fluharty Preschool Speech and Language Screening Tests. Pro-ed; 1978.

18. Brown R. A first language: the early stages. Cambridge: Harvard University Press; 1973.

19. Araujo K, Befi-Lopes DM. Extensão média do enunciado de crianças entre 2 e 4 anos de idade: diferenças no uso de palavras e morfemas. Rev Soc Bras Fonoaudiol. 2004;9: 156-63. 